\title{
Research on Grain System Evaluation Based on Fuzzy Evaluation System and GM(1,1) Method
}

\author{
Ruoyi Wang*, Mingzhi Xu, Biao Hou \\ Tianjin University of Finance and Economics, Tianjin, 300222, China \\ *Corresponding author
}

\begin{abstract}
With the development of the world, the current food problem has increasingly become the focus of international attention. Even today, in times of peace, the global food system is unstable. Part of the reason for these instability is our current global system of large-scale national and international food producers and distributors. In this paper, the relevant indexes of the relevant food system are collected, and a new food system evaluation system is established by using the mathematical model, and the optimization, discussion and analysis are carried out. In this paper, a new grain evaluation system is established by using fuzzy comprehensive evaluation method, and taking China as an example, the current natural and human factors in China are given and calculated, and finally get 70.64 points. Finally, it is found that China can realize the optimization of its food system only when the human development index reaches the United States today, and then understood by the grey prediction model, China will not be able to achieve it until 204120 years later.
\end{abstract}

Keywords: Grain system, Fuzzy evaluation, Index system, GM $(1,1)$

\section{Introduction}

In the world, although many countries have enough food, many people are still suffering from hunger, indicating problems with the global food system, distribution in distribution and instability[1]. Even in affluent areas, people are still hungry, and the number of hungry people in poor areas is even greater.

Moreover, the current food system leaves a massive environmental footprint accounting for " $29 \%$ of greenhouse gas emissions... up to $80 \%$ of biodiversity loss, $80 \%$ of deforestation, and $70 \%$ of all freshwater use." This indicates [2] that there are significant problems with the fairness and sustainability of our food system, so our team decided to analyze the current food system through mathematical modeling and hope to improve our food system through the results of the modelling [3].

\section{Construction of Comprehensive Evaluation Index system}

Because from low input and low efficiency of agricultural production to achieve the high investment, high benefit development, from the loose use to run fast, compact land use conversion process, not only need to transition from extensive cultivation to precision farming, it is more important to from a single isolation, using the technology of comprehensive and mutual coordination development [4]. It can be seen that the rational input of production factors such as light, temperature, water, soil, labor technology, policies, and capital has become the key to achieving a new level of food production and meeting the challenges of the market economy [5]. The structure of the comprehensive evaluation index system of the grain system is shown in Figure1.

The three-tier comprehensive evaluation index system [6] as shown in the figure, among them, $G_{1}, G_{2}, G_{3}, G_{4}, G_{5}, G_{6}$ represent different index subsets, and their specific meanings are as follows:

$G_{1}$ (Meteorological factors) $=\left\{\mathrm{g}_{11}, \mathrm{~g}_{12}, \mathrm{~g}_{13}\right\}=\{$ Irradiation; average monthly precipitation; sunshine time

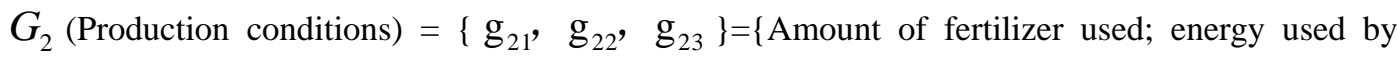
machinery; electricity used\} 
$G_{3}$ (Production level $)=\left\{\mathrm{g}_{31}, \mathrm{~g}_{32}\right\}=\{$ Yield per hectare; multiple cropping index $\}$
$G_{4}$ (Policy factors) $; G_{5}$ (International donation) $; G_{6}$ (Sustainability)

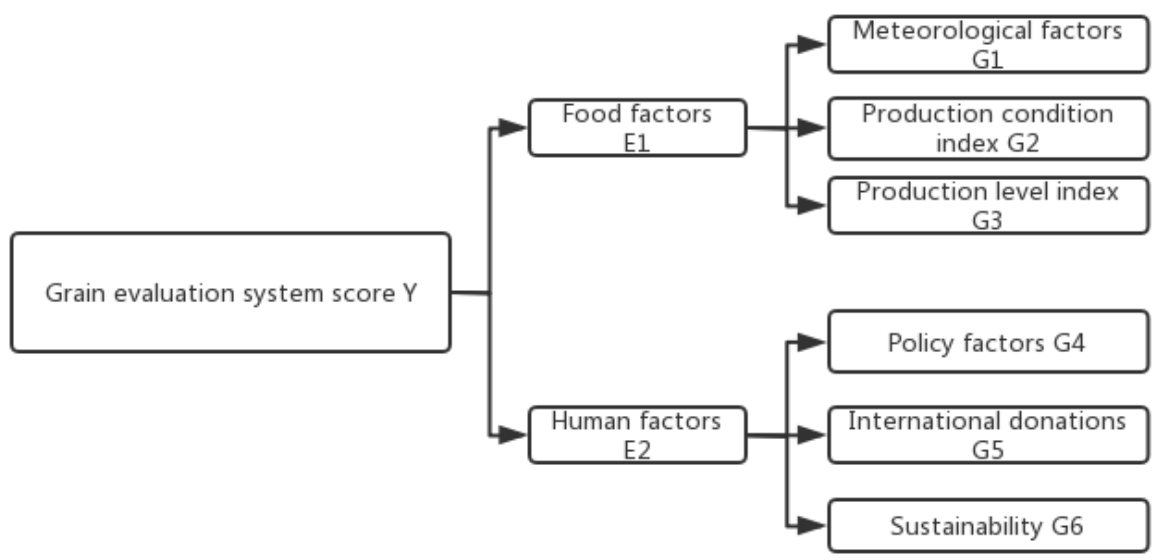

Figure 1: The comprehensive evaluation index system

\section{Fuzzy comprehensive evaluation}

\subsection{Model preparation}

The random survey method is used to determine the weight of each indicator layer. And take the evaluation comment $\mathrm{V}=\left\{v_{1}, v_{2}, v_{3}, v_{4}\right\}$, where $v_{1}, v_{2}, v_{3}, v_{4}$ respectively indicate that the indicators' comments are "excellent", "medium", "possible" and "poor", and the corresponding grain system rating is "excellent", "good", "medium" and "bad".

\subsection{Establishment of fuzzy evaluation model}

Fuzzy comprehensive evaluation for each $G_{i}(\mathrm{i}=1,2,3,4,5,6)$, if the indicators under $G_{i}$ $(\mathrm{i}=1,2,3,4,5,6)$ are considered separately $g_{i j}$, the degree to which $g_{i j}$ belongs to the kth comment $v_{t}$ can be obtained by random survey method. We can obtain the fuzzy evaluation matrix of A:

$$
R_{i}=\left(\begin{array}{cccc}
r_{i 11} & r_{i 12} & r_{i 13} & r_{i 14} \\
\cdots & \cdots & \cdots & \cdots \\
r_{i n 1} & r_{i n 2} & r_{i n 3} & r_{i n 4}
\end{array}\right)
$$

Where $\mathrm{n}$ is the number of indicators evaluated in $G_{i}$, since in terms of this model:

$$
G_{i}=W_{i} \circ R_{i}=\left(w_{1}, w_{2}, w_{3}\right) \circ\left(\begin{array}{cccc}
r_{i 11} & r_{i 12} & r_{i 13} & r_{i 14} \\
\ldots & \ldots & \ldots & \ldots \\
r_{i n 1} & r_{i n 2} & r_{i n 3} & r_{i n 4}
\end{array}\right)=\left(a_{i 1}, a_{i 2}, a_{i 3}, a_{i 4}\right)
$$

Obtain the fuzzy comprehensive evaluation set of each index of $\mathrm{G}$ layer, $A_{i}=\left(a_{1}, a_{2}, a_{3}, a_{4}\right)$, Where $w_{i}$ is the weight vector of the evaluation index in each $G_{i}, a_{i j}$ uses the $F(\bullet$,$) operator.$ Similarly, it can be obtained: 


$$
\begin{aligned}
& E_{1}=\left(p_{1}, p_{2}, p_{3}\right) \circ\left(\begin{array}{l}
G_{1} \\
G_{2} \\
G_{3}
\end{array}\right)=\left(e_{11}, e_{12}, e_{13}, e_{14}\right) \\
& E_{2}=\left(q_{1}, q_{2}, q_{3}\right) \circ\left(\begin{array}{l}
G_{4} \\
G_{5} \\
G_{6}
\end{array}\right)=\left(e_{21}, e_{22}, e_{23}, e_{24}\right)
\end{aligned}
$$

$p_{i}$ is the weight corresponding to each indicator $G_{i}(i=1,2,3), q_{i}$ weight corresponding to each weight of $G_{i}(i=4,5,6)$

\subsection{Determine the vector element set for evaluating the food system}

$$
Y=K \circ E=\left(k_{1}, k_{2}\right) \circ\left(\begin{array}{l}
E_{1} \\
E_{2}
\end{array}\right)=\left(y_{1}, y_{2}, y_{3}, y_{4}\right)
$$

Among them, $k_{i}$ is the weight vector corresponding to each $E_{i}(\mathrm{i}=1,2)$.

\section{Evaluation results based on weighted average}

\subsection{Evaluation results}

After quantifying the elements in the judgment set $\mathrm{V}$, the final judgment result $V=B . Y^{T}$. Where $B=\left(b_{1}, b_{2}, b_{3}, b_{4}\right)$ is the quantitative value of the four comments.

The specific calculation is as follows:

Taking China as an example, we are now using our current random survey method to obtain relevant data from China to obtain specific data on the indicator system of the food system. After that, we learned that China's donation ranking in the United Nations Food Program is 22. It can be seen that the overall international fairness is the upper-middle class and the relevant Chinese policies. Then we can empower and calculate:

Table 1: Calculation

\begin{tabular}{|c|c|c|c|}
\hline $\mathrm{K}=[0.4,0.6]$ & $\mathrm{P}=[0.3,0.3,0.4]$ & $\mathrm{Q}=[0.5,0.3,0.2]$ & \\
\hline $\mathrm{W} 1=[0.35,0.35,0.3]$ & $\mathrm{W} 2=[0.4,0.3,0.3]$ & $\mathrm{W} 3=[0.6,0.4]$ & \\
\hline $\mathrm{R} 1=[0.4,0.1,0.1,0.4$, & $\mathrm{R} 2=[0.3,0.2,0.2,0.3$, & $\mathrm{R} 3=[0.4,0.2,0.1,0.3$, & \\
$0.4,0.1,0.1,0.4$, & $0.3,0.25,0.15,0.3$, & $0.3,0.2,0.2,0.3]$ & \\
$0.4,0.1,0.1,0.4]$ & $0.35,0.1,0.1,0.35]$ & $\mathrm{G} 3=\mathrm{W} 3 * \mathrm{R} 3$ & $\mathrm{G} 4=[0.4,0.1,0.1,0.4]$ \\
\hline $\mathrm{G} 1=\mathrm{W} 1 * \mathrm{R} 1$ & $\mathrm{G} 2=\mathrm{W} 2 * \mathrm{R} 2$ & & \\
\hline $\mathrm{G} 5=[0.3,0.2,0.2,0.3]$ & $\mathrm{G} 6=[0.45,0.1,0.15,0.3]$ & & \\
\hline
\end{tabular}

If the quantitative value of each element in the evaluation set $\mathrm{V}$ is specified as $\mathrm{v} 1=100, \mathrm{v} 2=80, \mathrm{v} 3=60$, $\mathrm{v} 4=40$, the final evaluation result will be between 100 and 25 , usually close to 100 , the better the food system; the closer to 25, the worse. Then we used MATLAB to calculate and the final score was 70.64 points.

\subsection{System optimization}

The weight coefficients and evaluation grades in the above system of our food system evaluation can be virtual in the process of evaluation, but in the actual evaluation process, these data should be evaluated by special agencies and evaluation experts based on actual conditions. Then we will further refine these weights. According to our survey, the United States ranks 1 in the donation of the United Nations Food Program. According to the United States' attitude towards food donations, its international donation and 
sustainability will be better, and its policy impact the upper limit will be smaller, so we re-assign the weight of the food system evaluation:

Table 2: Optimized Calculatioin

\begin{tabular}{|c|c|c|c|}
\hline $\mathrm{K}=[0.6,0.4]$ & $\mathrm{P}=[0.3,0.3,0.4]$ & $\mathrm{Q}=[0.5,0.3,0.2]$ & \\
\hline $\mathrm{W} 1=0.35,0.35,0.3]$ & $\mathrm{W} 2=[0.4,0.3,0.3]$ & $\mathrm{W} 3=[0.6,0.4]$ & \\
\hline $\begin{array}{c}\mathrm{R} 1=0.4,0.1,0.1,0.4, \\
0.4,0.1,0.1,0.4, \\
0.4,0.1,0.1,0.4]\end{array}$ & $\begin{array}{c}\mathrm{R} 2=[0.3,0.2,0.2,0.3, \\
0.3,0.25,0.15,0.3, \\
0.35,0.1,0.1,0.35]\end{array}$ & $\begin{array}{c}\mathrm{R} 3=[4,0.2,0.1,0.3 \\
0.3,0.2,0.2,0.3]\end{array}$ & \\
\hline $\mathrm{G} 1=\mathrm{W} 1 * \mathrm{R} 1$ & $\mathrm{G} 2=\mathrm{W} 2 * \mathrm{R} 2$ & $\mathrm{G} 3=\mathrm{W} 3 * \mathrm{R} 3$ & $\mathrm{G} 4=[0.4,0.1,0.1,0.4]$ \\
\hline $\mathrm{G} 5=[0.65,0.2,0.1,0.05]$ & $\mathrm{G} 6=[0.6,0.2,0.1,0.1]$ & & \\
\hline
\end{tabular}

\section{GM $(1,1)$ grayscale prediction model}

Because the improved food system takes the United States as an example. It is well known that the United States is the representative of developed countries and China is the representative of developing countries. To achieve this level, taking China as an example, it must achieve the current United States.

That is, China's human development index can reach or exceed 0.926 before it can be achieved, so we will use the gray forecast model to predict.

We record the data of China from 2014 to 2019 as Matrix $A_{3 \times 2}$, which represents a total of 6 data in the third two years.

Calculate the average value of this quarter

$$
\mathrm{X}^{(0)}(i)=\frac{1}{2} \sum_{j=1}^{2} a_{i j} \quad \mathrm{i}=1,2,3
$$

The average value obtained is $\mathrm{X}^{(0)}=(0.735,1.4795,2.2375)$.

Generate value-added sequence:

$$
z^{(1)}(k)=\alpha x^{(1)}(k)+(1-\alpha) x^{(1)}(k-1) \mathrm{k}=2,3
$$

Here $\alpha=0.4$, in fact, generally $0 \leq \alpha \leq 1$.

Establish grey differential equation:

$$
x^{0}(k)+a z^{(1)}(k)=b, \mathrm{k}=2,3
$$

The corresponding $\operatorname{GM}(1,1)$ whitening differential equation is:

$$
\frac{d x^{(1)}}{d t}+a x^{(1)}(t)=b
$$

Then solve the differential equation to get $a$ and $b$.

Discrete solution of $\mathrm{GM}(1,1)$ model:

$$
\hat{x}^{(1)}(k)=\left[x^{(0)}(1)-\frac{b}{a}\right] e^{-a(k-1)}+\frac{b}{a}, \mathrm{k}=2,3
$$

The prediction model restored to the original series is:

$$
\widehat{x}^{(0)}(k)=\widehat{x}^{(1)}(k)-\widehat{x}^{(1)}(k-1), \mathrm{k}=2,3
$$

At this time, we performed multiple values and found that when $\mathrm{k}=14$, this time is the 14th two years, China's human development index is 0.920509 and 0.930852, that is, in 2040 and 2041, this data is $0.930852>0.926$ is currently 2021 . Taking China as an example, it will take 20 years. 


\section{Conclusion}

This paper focuses on the evaluation of the world food system, first establishes a fuzzy evaluation index system for evaluation and analysis, and takes China as an example to analyze and solve, and then uses the grayscale prediction model to study. Finally, it is concluded that the current natural and human factors in China are calculated, and the final score is 70.64. And China can only achieve the optimization of the food system when the human development index reaches the United States today, and only 20 years later in 2041 .

\section{References}

[1] MuHua Long, HongGuang Wang, YuTing Cui, DeXuan Li. Current situation and classification of grain production factors in China [J]. Crop magazine, 1994(01): 25-27.

[2] Efron, B. Bootstrap Method: Another Look at the Jackknife [J]. Annals of Statistics, 1979, 7(1): 126.

[3] ChengWei Sun, Jie Zhang, BaoTong Gu, ZhengHua Yu. Analysis of China's food security based on fuzzy comprehensive evaluation [J]. Shanxi Agricultural Economics, 2019(15): 2-4.

[4] LiZhi Cui. Grey prediction technology and its application [D]. Nanjing University of Aeronautics and Astronautics, 2010.

[5] MoYun Zhang, GuoAn Xiao. Research on improvement of grain yield prediction method in Hunan Province Based on Optimized Grey Model [J]. Journal of Xiangtan University (PHILOSOPHY AND SOCIAL SCIENCES EDITION), 2020, 44(03): 118-122.

[6] Yang H, Lin S. The Application of Improved Fuzzy Comprehensive Evaluation method in Practice Teaching Quality Evaluation System [J]. International Journal of Technology Management, 2013(8): 60-64. 